MATHEMATICS OF COMPUTATION

Volume 75 , Number 253 , Pages 151-165

S 0025-5718(05)01814-4

Article electronically published on September 15, 2005

\title{
STABILITY ANALYSIS \\ FOR DELAY DIFFERENTIAL EQUATIONS WITH MULTIDELAYS AND NUMERICAL EXAMPLES
}

\author{
LEPING SUN
}

ABstract. In this paper we are concerned with the asymptotic stability of the delay differential equation

$$
x^{\prime}(t)=A_{0} x(t)+\sum_{k=1}^{n} A_{k} x\left(t_{\tau_{k}}\right),
$$

where $A_{0}, A_{k} \in C^{d \times d}$ are constant complex matrices, and $x\left(t_{\tau_{k}}\right)=$ $\left(x_{1}\left(t-\tau_{k 1}\right), x_{2}\left(t-\tau_{k 2}\right), \ldots, x_{d}\left(t-\tau_{k d}\right)\right)^{T}, \tau_{k l}>0$ stand for $n \times d$ constant delays $(k=1, \ldots, n, l=1, \ldots, d)$. We obtain two criteria for stability through the evaluation of a harmonic function on the boundary of a certain region. We also get similar results for the neutral delay differential equation

$$
x^{\prime}(t)=L x(t)+\sum_{i=1}^{m} M_{i} x\left(t-\tau_{i}\right)+\sum_{j=1}^{n} N_{j} x^{\prime}\left(t-\tau_{j}^{\prime}\right),
$$

where $L, M_{i}$, and $N_{j} \in C^{d \times d}$ are constant complex matrices and $\tau_{i}, \tau_{j}^{\prime}>0$ stands for constant delays $(i=1, \ldots, m, j=1, \ldots, n)$. Numerical examples on various circumstances are shown to check our results which are more general than those already reported.

\section{INTRODUCTION}

Functional differential equations have a wide range of applications in science and engineering. The simplest and perhaps most natural type of functional differential equation is a "delay differential equation", that is, differential equations with dependence on the past state. The simplest type of past dependence is that it is carried through the state variable but not through its derivative. Then the equation can be expressed as delay differential equations (DDEs). There are also a number of applications in which the delayed argument occurs in the derivative of the state variables as well as in the state variable itself. Therefore, their models can be formulated with linear neutral delay differential equations (NDDEs)(see [9]).

Since analytical solutions of the above equations can be obtained only in very restricted cases, many methods have been proposed for the numerical approximation of the equations. At the same time, stability of numerical solutions is crucial in

Received by the editor March 2, 2003 and, in revised form, May 17, 2004.

2000 Mathematics Subject Classification. Primary 39A11.

Key words and phrases. Eigenvalue, matrix norm, spectral radius, boundary criteria, asymptotic stability, harmonic function, logarithmic norm.

The author was supported by the Shanghai Leading Academic Discipline Project, Project Number T0401.

(C)2005 American Mathematical Society Reverts to public domain 28 years from publication 
practical applications of DDEs and NDDEs. This numerical stability consideration is strongly supported by the analytical stability of the equations. Thus, the purpose of this paper is to study stability and numerical solutions of linear delay systems.

It is well known that the criteria for the stability of systems DDEs and NDDEs can be classified into two categories according to their dependence upon the size of delays. The criteria that do not include information on delays are refered to as the delay independent criteria [12, 13, 16, 17, 20, 21, 22, 23. Those carrying the information on delays are called the delay dependent criteria [12, 13, 17, 18, 19].

In $1996 \mathrm{Hu}$ [8, 9] discussed the stability and numerical solutions of the systems

$$
x^{\prime}(t)=\sum_{k=1}^{N} A_{k} x\left(t-\tau_{k}\right)
$$

and

$$
x^{\prime}(t)=A_{0} x(t)+\sum_{k=1}^{N} A_{k} x\left(t-\tau_{k}\right),
$$

where $x(t)$ is a function $R_{+} \rightarrow R^{n}$, and $A_{k} \in R^{n \times n}$ and $\tau_{k}$ denote $n$-square scalar matrices and any positive delays, respectively. He presented conditions of the delay independent stability and delay dependent stability for the system. As a special case of the system $x^{\prime}(t)=A_{0} x(t)+\sum_{k=1}^{N} A_{k} x\left(t-\tau_{k}\right)$,

$$
x^{\prime}(t)=A x(t)+B x(t-\tau)
$$

was discussed in 17. After that $\mathrm{Hu}$ and Mitsui [7 considered the asymptotic stability of systems of linear delay differential equations

$$
x^{\prime}(t)=A_{1} x(t)+A_{2} x(t-\tau),
$$

where $A_{1}$ and $A_{2}$ have $l(1 \leq l \leq n)$ common eigenvectors. A method for the analysis is presented by means of eigenpairs of the parameter matrices. They had the conclusion that under the condition that parameter matrices of the systems have common eigenvectors, the stability analysis may be reduced to that of lower dimensional systems. In $1991 \mathrm{Lu}$ [6] treated a simple but more interesting system of delay differential equations with double delays given by

$$
\begin{aligned}
& u^{\prime}(t)=a_{1} u(t)+b_{1} v\left(t-\tau_{2}\right), \\
& v^{\prime}(t)=a_{2} v(t)+b_{2} u\left(t-\tau_{1}\right),
\end{aligned}
$$

or in a vector form, by

$$
y^{\prime}(t)=A y(t)+B y\left(t_{\tau}\right),
$$

where matrices $A$ and $B$ are given by

$$
A=\left(\begin{array}{cc}
a_{1} & 0 \\
0 & a_{2}
\end{array}\right), \quad B=\left(\begin{array}{cc}
0 & b_{1} \\
b_{2} & 0
\end{array}\right)
$$

and the vectors mean

$$
y(t)=(u(t), v(t))^{T}, \quad y\left(t_{\tau}\right)=\left(u\left(t-\tau_{1}\right), v\left(t-\tau_{2}\right)\right)^{T} .
$$

He showed that the solution of the above equation satisfies the condition $\lim _{t \rightarrow \infty} y(t)=0$ if

$$
\operatorname{Re}\left(a_{i}\right)<0, \quad i=1,2, \quad \text { and } \quad\left|b_{1} b_{2}\right|<\operatorname{Re}\left(a_{1}\right) \operatorname{Re}\left(a_{2}\right) .
$$


In this paper we are concerned with the numerical solution and its stability of a more general system of DDEs (see [15])

$$
x^{\prime}(t)=A_{0} x(t)+\sum_{k=1}^{n} A_{k} x\left(t_{\tau_{k}}\right),
$$

where $A_{0}, A_{k} \in C^{d \times d}$ are constant complex matrices, and $x\left(t_{\tau_{k}}\right)=$ $\left(x_{1}\left(t-\tau_{k 1}\right), x_{2}\left(t-\tau_{k 2}\right), \ldots, x_{d}\left(t-\tau_{k d}\right)\right)^{T}, \tau_{k l}>0$ stand for $n \times d$ constant delays $(k=1, \ldots, n, l=1, \ldots, d)$. We shall study delay dependent criteria for the above system. Our stability criteria only require the evaluation of a real function on the boundary of a certain region in the complex plane. The region is given as the intersection of a rectangle and a half circle both specified with the system. We also get similar results for the linear NDDEs:

$$
x^{\prime}(t)=L x(t)+\sum_{i=1}^{m} M_{i} x\left(t-\tau_{i}\right)+\sum_{j=1}^{n} N_{j} x^{\prime}\left(t-\tau_{j}^{\prime}\right),
$$

where $L, M_{i}$, and $N_{j} \in C^{d \times d}$ are constant complex matrices and $\tau_{i}, \tau_{j}^{\prime}>0$ stands for constant delays $(i=1, \ldots, m, j=1, \ldots, n)$.

We will first introduce zeros of analytical function in a bounded region in Section 2 and the logarithmic norm of a matrix in Section 3. In Sections 4 and 5, stability of DDEs and NDDEs with multidelays are presented, respectively. In the last section, we give several numerical examples on various circumstances so as to check the results.

\section{ZEROS OF ANALYTICAL FUNCTIONS IN A BOUNDED REGION}

Let $T$ denote a bounded region of the complex plane. $\partial T$ and $\bar{T}$ represent boundary and closure of $T$, respectively. Then we have $\bar{T}=\partial T \cup T$. Let

$$
f(s)=f(x, y)=u(x, y)+i v(x, y),
$$

where $f(s)$ denotes an analytical function for $s \in \bar{T}$, and $i^{2}=-1, s=x+i y$, $u(x, y)=\operatorname{Re} f(s), v(x, y)=\operatorname{Im} f(s)$. The following two theorems give the sufficient conditions of the existence on nonzeros of $f(s)$ for any $s \in \bar{T}$.

Theorem 2.1 ([8]). If for any $(x, y) \in \partial T$ the real part $u(x, y)$ in $f(s)=f(x, y)=$ $u(x, y)+i v(x, y)$ does not vanish, then $f(x, y) \neq 0$ for any $(x, y) \in \bar{T}$.

Theorem $2.2(8)$. Assume that for any $(x, y) \in \partial T$ there exists a real constant $\lambda$ satisfying $u(x, y)+\lambda v(x, y) \neq 0$. Then $f(s)=u(x, y)+i v(x, y) \neq 0$ for any $(x, y) \in \bar{T}$.

Some researchers [5] have devoted their study to the determination of zeros of analytical functions in a bounded region.

\section{THE LOGARITHMIC NORM OF A MATRIX}

The measure of $A$ is defined by

$$
\lim _{\Delta \rightarrow 0} \frac{\|X+\Delta A\|-\|X\|}{\Delta}
$$

for any matrix norm and was introduced independently by Dahlquist 11] and Lozinskij [14. It has been applied extensively in the areas of numerical analysis and automatic control [3, 12]. 
The one-sided directional derivative of $\|\cdot\|$ at $I \in C^{n \times n}$ in the direction $A$ is called the logarithmic norm of the matrix $A$ and is denoted by $\mu(A)$. Thus,

$$
\mu(A)=\lim _{\Delta \rightarrow 0^{+}} \frac{\|I+\Delta A\|-1}{\Delta} .
$$

Theorem 3.1 (3]). Let $A \in C^{n \times n}$ and $\mu(A)=\lim _{\Delta \rightarrow 0^{+}} \frac{\|I+\Delta A\|-1}{\Delta}$. Then for each eigenvalue $\lambda$ of matrix $A \in C^{n \times n}$, the inequality

$$
-\mu(-A) \leq \operatorname{Re} \lambda_{i}(A) \leq \mu(A) \quad \forall i \in\{1,2, \ldots, n\}
$$

holds.

Theorem $3.2([3])$. Let $x=\left(x_{1}, x_{2}, \ldots, x_{n}\right)^{T} \in C^{n}$ and $A=\left(a_{i j}\right) \in C^{n \times n}$. Then

$$
\begin{aligned}
\mu_{1}(A) & =\max _{j}\left[\operatorname{Re}\left(a_{j j}\right)+\sum_{i, i \neq j}\left|a_{i j}\right|\right], \\
\mu_{2}(A) & =\frac{1}{2} \max _{i}\left[\lambda_{i}\left(A+A^{*}\right)\right], \\
\mu_{\infty}(A) & =\max _{i}\left[\operatorname{Re}\left(a_{i i}\right)+\sum_{j, j \neq i}\left|a_{i j}\right|\right] .
\end{aligned}
$$

The formulas of this theorem show that $\mu_{p}(A)$ is easy to calculate for $p=1, \infty$ or to estimate for $p=2$. In fact, $\mu_{p}(A)$ may actually be smaller than the corresponding matrix norm $\|A\|_{p}$. Also, $\mu_{p}(A)$ may be negative (see Section 6 ).

\section{Stability analysis of linear Delay differential equations (DDEs)}

4.1. Delay independent stability of DDEs with multidelays. Now we will deal with the asymptotic stability of DDEs

$$
x^{\prime}(t)=A_{0} x(t)+\sum_{k=1}^{n} A_{k} x\left(t_{\tau_{k}}\right)
$$

where $A_{0}, A_{k} \in C^{d \times d}$ are constant complex matrices, and $x\left(t_{\tau_{k}}\right)=$ $\left(x_{1}\left(t-\tau_{k 1}\right), x_{2}\left(t-\tau_{k 2}\right), \ldots, x_{d}\left(t-\tau_{k d}\right)\right)^{T}, \tau_{k l}>0(k=1, \ldots, n, l=1, \ldots, d)$ stand for constant delays.

For the stability of system (4.1), its characteristic equation

$$
\operatorname{det}\left[z I-A_{0}-\sum_{k=1}^{n} A_{k} \exp \left(-z T_{k}\right)\right]=0
$$

is crucial, where $\exp \left(-z T_{k}\right)=\operatorname{diag}\left\{e^{-z \tau_{k 1}}, \ldots, e^{-z \tau_{k d}}\right\}, k=1, \ldots, n$. The characteristic equation (4.2) is obtained from equation (4.1) by looking for nontrivial solutions of the form $x(t)=\xi \cdot e^{-z t}, \xi \in C^{d}$ [15]. The left hand side function of $z$ in (4.2) will be refered to the characteristic function, whose root is called a characteristic root of (4.2).

Definition. System (4.1) is said to be asymptotically stable if the solution $x(t)$ tends to zero as $t \rightarrow \infty$.

The following two lemmata are well known. 
Lemma 4.1 ([1]). If the real parts of all the characteristic roots of (4.2) are less than zero, then the system (4.1) is asymptotically stable.

For a square matrix $A,|A|$ stands for the matrix whose component is replaced by the modulus of the corresponding component of $A$, and $\rho(A)$ means the spectral radius of $A$ which is the greatest modulus value of eigenvalues of a matrix.

Lemma 4.2 ([4]). Let $A \in C^{d \times d}$ and $B \in R^{d \times d}$. If the inequality $|A| \leq B$ holds, then the inequality $\rho(A) \leq \rho(B)$ is valid. Here the order relation of matrices of the same dimensions should be interpreted componentwise.

For a complex matrix $W$, let $\mu(W)$ be the logarithmic norm of $W$ and

$$
\mu(W)=\lim _{\Delta \rightarrow 0^{+}} \frac{\|I+\Delta W\|-1}{\Delta} .
$$

Note that $\mu(W)$ depends on the chosen matrix norm. Let $\|W\|$ denote the matrix norm of $W$ subordinate to a certain vector norm. In order to specify the norm, the notation $\|\cdot\|_{p}$ is used. And the notation $\mu_{p}(\cdot)$ is also adopted to denote the logarithmic norm associated with $\|\cdot\|_{p}$, where $p=1,2, \infty$.

The characteristic function of (4.2) may be written as

$$
P(z)=\operatorname{det}\left[z I-A_{0}-\sum_{k=1}^{n} A_{k} \exp \left(-z T_{k}\right)\right]=U(x, y)+i V(x, y),
$$

where $z=x+i y$.

The following lemma states a sufficient condition for the delay independent stability of (4.1).

Lemma 4.3. If the condition

$$
\mu\left(A_{0}\right)+\sum_{k=1}^{n}\left\|A_{k}\right\|<0
$$

holds, then the system (4.1) is asymptotically stable.

Proof. Assume that the condition of the lemma is satisfied and that the system (4.1) is unstable. There is a root $z$ of $P(z)$ satisfying $\operatorname{Re}(z) \geq 0$.

Note that $z$ is also an eigenvalue of the matrix $A_{0}+\sum_{k=1}^{n} A_{k} e^{-z T_{k}}$. Applying the properties of the logarithmic norm and theorem in Section 3, we have the inequalities

$$
\begin{aligned}
0 & \leq \operatorname{Re}(z) \leq \mu\left(A_{0}+\sum_{k=1}^{n} A_{k} e^{-z T_{k}}\right)=\lim _{\Delta \rightarrow 0^{+}} \frac{\left\|I+\Delta\left(A_{0}+\sum_{k=1}^{n} A_{k} e^{-z T_{k}}\right)\right\|-1}{\Delta} \\
& \leq \mu\left(A_{0}\right)+\sum_{k=1}^{n}\left\|A_{k}\right\| \max _{1 \leq l \leq d}\left|e^{-z \tau_{k l}}\right| \leq \mu\left(A_{0}\right)+\sum_{k=1}^{n}\left\|A_{k}\right\| .
\end{aligned}
$$

This, however, contradicts condition (4.4). The proof of the lemma is completed.

From the above lemma, if $\mu\left(A_{0}\right)+\sum_{k=1}^{n}\left\|A_{k}\right\| \geq 0$, system (4.1) may be stable or unstable. The following theorem gives a region including all the roots of (4.2) with nonnegative real parts when the condition of the lemma fails. 
Theorem 4.4. Suppose that there exists a root of (4.2) whose real part is nonnegative.

(i) If we have the inequality

$$
\mu\left(A_{0}\right)+\sum_{k=1}^{n}\left\|A_{k}\right\|>0
$$

then the inequalities

$$
0 \leq \operatorname{Re}(z) \leq \mu\left(A_{0}\right)+\sum_{k=1}^{n}\left\|A_{k}\right\|
$$

and

$$
-\mu\left(i A_{0}\right)-\sum_{k=1}^{n}\left\|A_{k}\right\| \leq \operatorname{Im}(z) \leq \mu\left(-i A_{0}\right)+\sum_{k=1}^{n}\left\|A_{k}\right\|
$$

hold.

(ii) If we have the inequality

$$
-\mu\left(-A_{0}\right)-\sum_{k=1}^{n}\left\|A_{k}\right\|>0
$$

and if $\beta$ denotes a positive number satisfying

$$
-\mu\left(-A_{0}\right)-\sum_{k=1}^{n}\left\|A_{k}\right\| \max _{1 \leq l \leq d} e^{-\beta \tau_{k l}}=\beta,
$$

then the inequalities

$$
\beta \leq \operatorname{Re}(z) \leq \mu\left(A_{0}\right)+\sum_{k=1}^{n}\left\|A_{k}\right\| \max _{1 \leq l \leq d} e^{-\beta \tau_{k l}}
$$

and

$$
-\mu\left(i A_{0}\right)-\sum_{k=1}^{n}\left\|A_{k}\right\| \max _{1 \leq l \leq d} e^{-\beta \tau_{k l}} \leq \operatorname{Im}(z) \leq \mu\left(-i A_{0}\right)+\sum_{k=1}^{n}\left\|A_{k}\right\| \max _{1 \leq l \leq d} e^{-\beta \tau_{k l}}
$$

are valid.

Proof. (i) A proof similar to that of Lemma 4.3 yields

$$
0 \leq \operatorname{Re}(z) \leq \mu\left(A_{0}\right)+\sum_{k=1}^{n}\left\|A_{k}\right\| .
$$

Next, the imaginary part of an eigenvalue of a matrix $A$ is equal to the real part of the eigenvalue of $-i A$. Therefore, the second inequality $-\mu\left(i A_{0}\right)-\sum_{k=1}^{n}\left\|A_{k}\right\| \leq$ $\operatorname{Im}(z) \leq \mu\left(-i A_{0}\right)+\sum_{k=1}^{n}\left\|A_{k}\right\|$ holds.

(ii) By Theorem 3.1,

$$
-\mu\left(-A_{0}-\sum_{k=1}^{n} A_{k} e^{-z T_{k}}\right) \leq \operatorname{Re}(z) \leq \mu\left(A_{0}+\sum_{k=1}^{n} A_{k} e^{-z T_{k}}\right) .
$$

A similar derivation to that in Lemma 4.3 and (i) leads to

$$
-\mu\left(-A_{0}\right)-\sum_{k=1}^{n}\left\|A_{k}\right\| \leq \operatorname{Re}(z) \leq \mu\left(A_{0}\right)+\sum_{k=1}^{n}\left\|A_{k}\right\| \max _{1 \leq l \leq d}\left(e^{-\operatorname{Re}(z) \cdot \tau_{k l}}\right) .
$$


Set

$$
\beta_{0}=-\mu\left(-A_{0}\right)-\sum_{k=1}^{n}\left\|A_{k}\right\|
$$

By (4.5),

$$
\begin{aligned}
\operatorname{Re}(z) & \geq-\mu\left(-A_{0}-\sum_{k=1}^{n} A_{k} e^{-z T_{k}}\right) \geq-\mu\left(-A_{0}\right)-\sum_{k=1}^{n}\left\|A_{k}\right\| \max _{1 \leq l \leq d}\left|e^{-z \tau_{k l}}\right| \\
& =-\mu\left(-A_{0}\right)-\sum_{k=1}^{n}\left\|A_{k}\right\| \max _{1 \leq l \leq d}\left(e^{-\operatorname{Re}(z) \cdot \tau_{k l}}\right) .
\end{aligned}
$$

Henceforth, together with (4.6),

$$
\operatorname{Re}(z) \geq-\mu\left(-A_{0}\right)-\sum_{k=1}^{n}\left\|A_{k}\right\| \max _{1 \leq l \leq d}\left(e^{-\beta_{0} \tau_{k l}}\right) .
$$

Let $\beta_{1}=-\mu\left(-A_{0}\right)-\sum_{k=1}^{n}\left\|A_{k}\right\| \max _{1 \leq l \leq d}\left(e^{-\beta_{0} \tau_{k l}}\right)$. Then we have

$$
\operatorname{Re}(z) \geq \beta_{1} \geq \beta_{0} .
$$

Let $\beta_{2}=-\mu\left(-A_{0}\right)-\sum_{k=1}^{n}\left\|A_{k}\right\| \max _{1 \leq l \leq d}\left(e^{-\beta_{1} \tau_{k l}}\right)$. We again have

$$
\operatorname{Re}(z) \geq \beta_{2} \geq \beta_{1} \geq \beta_{0} .
$$

The iteration

$$
-\mu\left(-A_{0}\right)-\sum_{k=1}^{n}\left\|A_{k}\right\| \max _{1 \leq l \leq d}\left(e^{-\beta_{j} \tau_{k l}}\right)=\beta_{j+1} \geq \beta_{j},(j=0,1, \ldots,)
$$

and the monotonicity

$$
\beta_{0} \leq \beta_{1} \leq \cdots \leq \beta_{j} \leq \beta_{j+1} \leq \cdots \leq \operatorname{Re}(z) \leq \mu\left(A_{0}\right)+\sum_{k=1}^{n}\left\|A_{k}\right\|
$$

assure that the limit of the series $\left\{\beta_{j}\right\}$ exists and is equal to $\beta$, where $\beta$ is a positive number satisfying

$$
-\mu\left(-A_{0}\right)-\sum_{k=1}^{n}\left\|A_{k}\right\| \max _{1 \leq l \leq d} e^{-\beta \tau_{k l}}=\beta .
$$

Therefore, the first inequality holds. Next, the imaginary part of an eigenvalue of a matrix $A$ is equal to the real part of the eigenvalue of $-i A$. Therefore, the second inequality

$$
-\mu\left(i A_{0}\right)-\sum_{k=1}^{n}\left\|A_{k}\right\| \max _{1 \leq l \leq d} e^{-\beta \tau_{k l}} \leq \operatorname{Im}(z) \leq \mu\left(-i A_{0}\right)+\sum_{k=1}^{n}\left\|A_{k}\right\| \max _{1 \leq l \leq d} e^{-\beta \tau_{k l}}
$$

holds.

In what follows, $\lambda_{j}(A)(j=1,2, \ldots, d)$ stands for the $j$ th eigenvalue of $A \in C^{d \times d}$.

Theorem 4.5. If $z$ is a characteristic root of (4.2) with nonnegative real part, then the inequality

$$
|z| \leq \rho\left(\left|A_{k}\right|+\sum_{k=1}^{n}\left|A_{k}\right|\right)
$$

holds. 
Proof. By the assumption above, there exists an integer $j(1 \leq j \leq d)$ such that

$$
z=\lambda_{j}\left(A_{0}+\sum_{k=1}^{n} A_{k} e^{-z T_{k}}\right) .
$$

This implies the inequality

$$
|z| \leq \rho\left(A_{0}+\sum_{k=1}^{n} A_{k} e^{-z T_{k}}\right) .
$$

It is obvious that

$$
\left|A_{0}+\sum_{k=1}^{n} A_{k} e^{-z T_{k}}\right| \leq\left|A_{0}\right|+\sum_{k=1}^{n}\left|A_{k}\right| \cdot\left|e^{-z T_{k}}\right| \leq\left|A_{0}\right|+\sum_{k=1}^{n}\left|A_{k}\right| .
$$

Therefore, due to Lemma 4.2, we have the conclusion.

\subsection{Delay dependent stability of DDEs. Let}

$$
\gamma=\mu\left(A_{0}\right)+\sum_{k=1}^{n}\left\|A_{k}\right\|
$$

By virtue of Lemma 4.3, if $\gamma<0$, the system (4.1) is delay independent asymptotically stable. If $\gamma \geq 0$, the system (4.1) may be stable or unstable. In this section, we discuss the stability of (4.1) when $\gamma \geq 0$.

Let $\beta_{0}=-\mu\left(-A_{0}\right)-\sum_{k=1}^{n}\left\|A_{k}\right\|$ and $\gamma=\mu\left(A_{0}\right)+\sum_{k=1}^{n}\left\|A_{k}\right\|$ and $\gamma \geq 0$. We define the following quantities according to the sign of $\beta$ (see Theorem 4.4).

(i) If $\beta_{0} \leq 0$, then we put

$$
\begin{gathered}
E_{0}=0, \quad E=\mu\left(A_{0}\right)+\sum_{k=1}^{n}\left\|A_{k}\right\|, \\
F_{0}=-\mu\left(i A_{0}\right)-\sum_{k=1}^{n}\left\|A_{k}\right\|, \quad \text { and } F=\mu\left(-i A_{0}\right)+\sum_{k=1}^{n}\left\|A_{k}\right\| .
\end{gathered}
$$

(ii) If $\beta_{0}>0$, then we put

$$
\begin{gathered}
E_{0}=\beta, \quad E=\mu\left(A_{0}\right)+\sum_{k=1}^{n}\left\|A_{k}\right\| \max _{1 \leq l \leq d} e^{-\beta \tau_{k l}}, \\
F_{0}=-\mu\left(i A_{0}\right)-\sum_{k=1}^{n}\left\|A_{k}\right\| \max _{1 \leq l \leq d} e^{-\beta \tau_{k l}} \\
F=\mu\left(-i A_{0}\right)+\sum_{k=1}^{n}\left\|A_{k}\right\| \max _{1 \leq l \leq d} e^{-\beta \tau_{k l}}, \text { where } \beta \text { is a root of the equation } \\
-\mu\left(-A_{0}\right)-\sum_{k=1}^{n}\left\|A_{k}\right\| \max _{1 \leq l \leq d} e^{-\beta \tau_{k l}}=\beta .
\end{gathered}
$$

Under the above notation we turn our attention to the following three kinds of bounded regions in the $z$-plane.

Definition 4.6. Let $l_{1}, l_{2}, l_{3}$, and $l_{4}$ denote the segments $\left\{\left(E_{0}, y\right): F_{0} \leq y \leq F\right\}$, $\left\{(x, F): E_{0} \leq x \leq E\right\},\left\{(E, y): F_{0} \leq y \leq F\right\}$, and $\left\{\left(x, F_{0}\right): E_{0} \leq x \leq E\right\}$, respectively. Furthermore, let $l=l_{1} \cup l_{2} \cup l_{3} \cup l_{4}$ and $D$ be the rectangular region surrounded by $l$. 
Definition 4.7. Let $R=\rho\left(\left|A_{k}\right|+\sum_{k=1}^{n}\left|A_{k}\right|\right)$. Let $K$ denote the circular region with radius $R$ centered at the origin in the plane of $C$,

$$
K=\{(r, \theta): r \leq R, 0 \leq \theta \leq 2 \pi\} .
$$

Definition 4.8. Let $D$ be as defined in Definition 4.6, and let $T$ represent the intersection $D \cap K$. The boundary of $T$ is denoted by $\partial T$ and $\bar{T}=T \cup \partial T$.

The following two theorems establish criteria for the delay dependent stability of DDEs (4.1). We apply Theorems 2.1 and 2.2 to prove them, respectively.

The first boundary criterion of stability is as follows.

Theorem 4.9. If for any $(x, y) \in \partial T$, the real part $U(x, y)$ in (4.3) does not vanish, then the system (4.1) is asymptotically stable.

Proof. Assume that the condition is satisfied and that the system (4.1) is unstable. This implies the existence of a characteristic root $z$ of (4.2) with a nonnegative real part. According to Lemma 4.1, it suffices to prove that $P(z) \neq 0$ for $\operatorname{Re}(z) \geq 0$. Applying Theorems 4.4 and 4.5 and Definition 4.8, it is sufficient to consider for $z \in \bar{T}$.

From the assumption of this theorem and the statement of Theorem 2.1, this contradicts with $P(z)=0$ for $z \in \bar{T}$. Hence $P(z) \neq 0$ for $\operatorname{Re}(z) \geq 0$ and the proof is completed.

Due to Theorem 2.2, we can further extend the above result as follows.

Theorem 4.10. Assume that for any $(x, y) \in \partial T$, there exists a real constant $\lambda$ satisfying

$$
U(x, y)+\lambda V(x, y) \neq 0 .
$$

Then the system (4.1) is asymptotically stable.

The proof is analogous to Theorem 4.9 .

\section{Stability analysis of NDDEs}

5.1. Delay independent stability of NDDEs with multidelays. Now we deal with the asymptotic stability of NDDEs,

$$
x^{\prime}(t)=L x(t)+\sum_{i=1}^{m} M_{i} x\left(t-\tau_{i}\right)+\sum_{j=1}^{n} N_{j} x^{\prime}\left(t-\tau_{j}^{\prime}\right),
$$

where $L, M_{i}$, and $N_{j} \in C^{d \times d}$ are constant complex matrices and $\tau_{i}, \tau_{j}>0$ stands for constant delays $(i=1, \ldots, m, j=1, \ldots, n)$.

For the stability of the system (5.1), we investigate its characteristic equation

$$
P(z)=\operatorname{det}\left[z I-L-\sum_{i=1}^{m} M_{i} e^{-z \tau_{i}}-z \sum_{j=1}^{n} N_{j} e^{-z \tau_{j}^{\prime}}\right]=0,
$$

where $z$ is a root of the equation.

The above characteristic equation (5.2) may be written as

$$
\operatorname{det}\left[z I-L-\sum_{i=1}^{m} M_{i} e^{-z \tau_{i}}-z \sum_{j=1}^{n} N_{j} e^{-z \tau_{j}^{\prime}}\right]=U(x, y)+i V(x, y),
$$

where $z=x+i y$. 
Definition. The solution of (5.1) is said to be asymptotically stable if the solution $x(t)$ tends to zero as $t \rightarrow \infty$.

Lemma 5.1. Let $\sum_{j=1}^{n}\left\|N_{j}\right\|<1$. If the condition

$$
\mu(L)+\frac{\sum_{i=1}^{m}\left\|M_{i}\right\|+\|L\| \cdot \sum_{j=1}^{n}\left\|N_{j}\right\|}{1-\sum_{j=1}^{n}\left\|N_{j}\right\|}<0
$$

holds, then the system (5.1) is asymptotically stable.

Proof. Assume that the condition of the lemma is satisfied and that the system (5.1) is unstable. There is a root of $P(z)$ satisfying $\operatorname{Re}(z) \geq 0$. Note that $z$ is also an eigenvalue of the matrix $L+\sum_{i=1}^{m} M_{i} e^{-z \tau_{i}}+z \sum_{j=1}^{n} N_{j} e^{-z \tau_{j}^{\prime}}$. The inner product

$$
z=\langle L x, x\rangle+\sum_{i=1}^{m}\left\langle M_{i} x, x\right\rangle e^{-z \tau_{i}}+z \sum_{j=1}^{n}\left\langle N_{j} x, x\right\rangle e^{-z \tau_{j}},
$$

where $x \in C^{d},\|x\|=1$, implies

$$
\begin{gathered}
|z| \leq\|L\|+\sum_{i=1}^{m}\left\|M_{i}\right\|+|z| \cdot \sum_{j=1}^{n}\left\|N_{j}\right\|, \quad \text { or } \\
|z| \leq \frac{\|L\|+\sum_{i=1}^{m}\left\|M_{i}\right\|}{1-\sum_{j=1}^{n}\left\|N_{j}\right\|} .
\end{gathered}
$$

Applying the properties of the logarithmic norm and Theorem 3.1, we have the inequalities

$$
\begin{aligned}
0 & \leq \operatorname{Re}(z) \leq \mu\left(L+\sum_{i=1}^{m} M_{i} e^{-z \tau_{i}}+z \sum_{j=1}^{n} N_{j} e^{-z \tau_{j}^{\prime}}\right) \\
& \leq \mu(L)+\sum_{i=1}^{m}\left\|M_{i}\right\|+|z| \cdot \sum_{j=1}^{n}\left\|N_{j}\right\| \\
& \leq \mu(L)+\frac{\sum_{i=1}^{m}\left\|M_{i}\right\|+\|L\| \cdot \sum_{j=1}^{n}\left\|N_{j}\right\|}{1-\sum_{j=1}^{n}\left\|N_{j}\right\|} .
\end{aligned}
$$

This, however, contradicts the condition (5.4). Hence the proof is completed.

The following Theorem 5.2 gives a region including all the roots of (5.2) with nonnegative real parts when the condition of Lemma 5.1 fails.

Theorem 5.2. Let $\sum_{j=1}^{n}\left\|N_{j}\right\|<1$. Suppose that there exists a root of (5.2) whose real part is nonnegative.

(i) If we have the inequality

$$
\mu(L)+\frac{\sum_{i=1}^{m}\left\|M_{i}\right\|+\|L\| \cdot \sum_{j=1}^{n}\left\|N_{j}\right\|}{1-\sum_{j=1}^{n}\left\|N_{j}\right\|}>0,
$$

then the inequalities

$$
0 \leq \operatorname{Re}(z) \leq \mu(L)+\frac{\sum_{i=1}^{m}\left\|M_{i}\right\|+\|L\| \cdot \sum_{j=1}^{n}\left\|N_{j}\right\|}{1-\sum_{j=1}^{n}\left\|N_{j}\right\|}
$$


and

$$
\begin{aligned}
& -\mu(i L)-\frac{\sum_{i=1}^{m}\left\|M_{i}\right\|+\|L\| \cdot \sum_{j=1}^{n}\left\|N_{j}\right\|}{1-\sum_{j=1}^{n}\left\|N_{j}\right\|} \\
& \quad \leq \operatorname{Im}(z) \leq \mu(-i L)+\frac{\sum_{i=1}^{m}\left\|M_{i}\right\|+\|L\| \cdot \sum_{j=1}^{n}\left\|N_{j}\right\|}{1-\sum_{j=1}^{n}\left\|N_{j}\right\|}
\end{aligned}
$$

hold.

(ii) If we have the inequality

$$
-\mu(-L)-\frac{\sum_{i=1}^{m}\left\|M_{i}\right\|+\|L\| \cdot \sum_{j=1}^{n}\left\|N_{j}\right\|}{1-\sum_{j=1}^{n}\left\|N_{j}\right\|}>0
$$

and if $\beta$ denotes a positive number satisfying

$$
-\mu(-L)-\frac{\sum_{i=1}^{m}\left\|M_{i}\right\|+\|L\| \cdot \sum_{j=1}^{n}\left\|N_{j}\right\|}{1-\sum_{j=1}^{n}\left\|N_{j}\right\|} e^{-\beta \tau}=\beta,
$$

then the inequalities

$$
\beta \leq \operatorname{Re}(z) \leq \mu(L)+\frac{\sum_{i=1}^{m}\left\|M_{i}\right\|+\|L\| \cdot \sum_{j=1}^{n}\left\|N_{j}\right\|}{1-\sum_{j=1}^{n}\left\|N_{j}\right\|} e^{-\beta \tau}
$$

and

$$
\begin{aligned}
& -\mu(i L)-\frac{\sum_{i=1}^{m}\left\|M_{i}\right\|+\|L\| \cdot \sum_{j=1}^{n}\left\|N_{j}\right\|}{1-\sum_{j=1}^{n}\left\|N_{j}\right\|} e^{-\beta \tau} \\
& \quad \leq \operatorname{Im}(z) \leq \mu(-i L)+\frac{\sum_{i=1}^{m}\left\|M_{i}\right\|+\|L\| \cdot \sum_{j=1}^{n}\left\|N_{j}\right\|}{1-\sum_{j=1}^{n}\left\|N_{j}\right\|} e^{-\beta \tau}
\end{aligned}
$$

are valid. Here $\tau=\min \left(\tau_{1}, \ldots, \tau_{m}, \tau_{1}^{\prime}, \ldots, \tau_{n}^{\prime}\right)$.

The proof is analogous to Theorem 4.4 with $\tau=\min \left(\tau_{1}, \ldots, \tau_{m}, \tau_{1}^{\prime}, \ldots, \tau_{n}^{\prime}\right)$.

Theorem 5.3. Let $\sum_{j=1}^{n}\left\|N_{j}\right\|<1$. If $z$ is a characteristic root of (5.2) with a nonnegative real part, then the inequality

$$
|z| \leq \rho\left(|L|+\sum_{i=1}^{m}\left|M_{i}\right|+\frac{\|L\|+\sum_{i=1}^{m}\left\|M_{i}\right\|}{1-\sum_{j=1}^{n}\left\|N_{j}\right\|} \cdot \sum_{j=1}^{n}\left|N_{j}\right|\right)
$$

holds.

The proof is analogous to Theorem 4.5 .

\subsection{Delay dependent stability of NDDEs. Let}

$$
\gamma=\mu(L)+\frac{\sum_{i=1}^{m}\left\|M_{i}\right\|+\|L\| \cdot \sum_{j=1}^{n}\left\|N_{j}\right\|}{1-\sum_{j=1}^{n}\left\|N_{j}\right\|} .
$$

By virtue of Lemma 5.1 if $\gamma<0$, the system (5.1) is delay independent asymptotically stable. If $\gamma \geq 0$, the system (5.1) may be stable or unstable. We consider the stability of (5.1) when $\gamma \geq 0$.

Let $\beta_{0}=-\mu(-L)-\frac{\sum_{i=1}^{m}\left\|M_{i}\right\|+\|L\| \cdot \sum_{j=1}^{n}\left\|N_{j}\right\|}{1-\sum_{j=1}^{n}\left\|N_{j}\right\|}$ and $\gamma \geq 0$. We define the following quantities according to the sign of $\beta$ (see Theorem 5.2). 
(i) If $\beta_{0} \leq 0$, then we put

$$
\begin{aligned}
E_{0} & =0, E=\mu(L)+\frac{\sum_{i=1}^{m}\left\|M_{i}\right\|+\|L\| \cdot \sum_{j=1}^{n}\left\|N_{j}\right\|}{1-\sum_{j=1}^{n}\left\|N_{j}\right\|}, \\
F_{0} & =-\mu(i L)-\frac{\sum_{i=1}^{m}\left\|M_{i}\right\|+\|L\| \cdot \sum_{j=1}^{n}\left\|N_{j}\right\|}{1-\sum_{j=1}^{n}\left\|N_{j}\right\|}, \\
F & =\mu(-i L)+\frac{\sum_{i=1}^{m}\left\|M_{i}\right\|+\|L\| \cdot \sum_{j=1}^{n}\left\|N_{j}\right\|}{1-\sum_{j=1}^{n}\left\|N_{j}\right\|} .
\end{aligned}
$$

(ii) If $\beta_{0}>0$, then we put

$$
\begin{aligned}
& E_{0}=\beta, E=\mu(L)+\frac{\sum_{i=1}^{m}\left\|M_{i}\right\|+\|L\| \cdot \sum_{j=1}^{n}\left\|N_{j}\right\|}{1-\sum_{j=1}^{n}\left\|N_{j}\right\|} e^{-\beta \tau}, \\
& F_{0}=-\mu(i L)-\frac{\sum_{i=1}^{m}\left\|M_{i}\right\|+\|L\| \cdot \sum_{j=1}^{n}\left\|N_{j}\right\|}{1-\sum_{j=1}^{n}\left\|N_{j}\right\|} e^{-\beta \tau}, \\
& F=\mu(-i L)+\frac{\sum_{i=1}^{m}\left\|M_{i}\right\|+\|L\| \cdot \sum_{j=1}^{n}\left\|N_{j}\right\|}{1-\sum_{j=1}^{n}\left\|N_{j}\right\|} e^{-\beta \tau},
\end{aligned}
$$

where $\beta$ is a root of the equation

$$
-\mu(-L)-\frac{\sum_{i=1}^{m}\left\|M_{i}\right\|+\|L\| \cdot \sum_{j=1}^{n}\left\|N_{j}\right\|}{1-\sum_{j=1}^{n}\left\|N_{j}\right\|} e^{-\beta \tau}=\beta,
$$

and $\tau=\min \left(\tau_{1}, \ldots, \tau_{m}, \tau_{1}^{\prime}, \ldots, \tau_{n}^{\prime}\right)$.

Under the above notation we turn our attention to the following three kinds of bounded regions in the $z$-plane.

Definition 5.4. Let $l_{1}, l_{2}, l_{3}$, and $l_{4}$ denote the segments $\left\{\left(E_{0}, y\right): F_{0} \leq y \leq F\right\}$, $\left\{(x, F): E_{0} \leq x \leq E\right\},\left\{(E, y): F_{0} \leq y \leq F\right\}$, and $\left\{\left(x, F_{0}\right): E_{0} \leq x \leq E\right\}$, respectively. Furthermore, let $l=l_{1} \cup l_{2} \cup l_{3} \cup l_{4}$ and $D$ be the rectangular region surrounded by $l$.

Definition 5.5. Let $R=\rho\left(|L|+\sum_{i=1}^{m}\left|M_{i}\right|+\frac{\|L\|+\sum_{i=1}^{m}\left\|M_{i}\right\|}{1-\sum_{j=1}^{n}\left\|N_{j}\right\|} \cdot \sum_{j=1}^{n}\left|N_{j}\right|\right)$. Let $K$ denote the circular region with radius $R$ centered at the origin in the plane of $C$,

$$
K=\{(r, \theta): r \leq R, 0 \leq \theta \leq 2 \pi\} .
$$

Definition 5.6. Let $T$ represent the intersection $D \cap K$. The boundary of $T$ is denoted by $\partial T$ and $\bar{T}=T \cup \partial T$.

The following two theorems give criteria for NDDEs (5.1).

Theorem 5.7. If for any $(x, y) \in \partial T$, the real part $U(x, y)$ in (5.3) does not vanish, then the system (5.1) is asymptotically stable.

The proof is analogous to Theorem 4.9.

Theorem 5.8. Assume that for any $(x, y) \in \partial T$, there exists a real constant $\lambda$ satisfying

$$
U(x, y)+\lambda V(x, y) \neq 0 .
$$

Then the system (5.1) is asymptotically stable.

The proof is analogous to Theorem 4.9. 


\section{NUMERICAL EXAMPLES}

In Section 6, we give two criteria for delay dependent stability of the linear delay system (4.1). Theorems 4.4 and 4.5 show that unstable characteristic roots of the system (4.1) are located in some specified bounded region of the complex plane, while Theorems 4.9 and 4.10 show that it is sufficient to check certain conditions on its boundary to exclude the possibility of such roots from the region. Theorems 2.1 and 2.2 generally provide simple criteria for nonexistence of zeros of an analytic function in any bounded region. In Section 5, we get the same results for the stability of linear neutral delay differential equations, system (5.1). In this section we list several numerical examples on various circumstances. These examples locate regions enclosing characteristic roots with positive real parts.

In the following, the first two examples are on system (4.1) by taking $k=1$, and the third one is on system (5.1) by taking $i=j=1, \tau \neq \tau^{\prime}$. In the following, $\mu(\cdot)$ denotes $\mu_{2}(\cdot)$, and $\|\cdot\|$ denotes $\|\cdot\|_{2}$. All the matrices are created randomly by the MATLAB program.

Example 1. Let

$$
A=\left(\begin{array}{lll}
.9501 & .4806 & .4565 \\
.2311 & .8913 & .0185 \\
.6068 & .7621 & .8214
\end{array}\right), \quad \text { and } \quad B=\left(\begin{array}{ccc}
.4447 & .9218 & .4057 \\
.6154 & .7382 & .9355 \\
.7919 & .1763 & .9169
\end{array}\right)
$$

According to Section 3, Theorems 4.4, 4.5, and Definitions 4.6 and 4.7, we get

$$
\begin{aligned}
\mu(A) & =1.74695, \mu(-A)=-0.3402, \mu(i A)=\mu(-i A)=0.40015,\|B\|=2.0069 \\
\gamma & =3.75385>0, \beta_{0}=-1.6667<0, R=3.7098
\end{aligned}
$$

So we take

$$
E_{0}=0, E=3.75385, F_{0}=-2.40705, F=2.40705 .
$$

Let $\tau_{1}=1, \tau_{2}=2, \tau_{3}=3$, respectively. Then the characteristic equation is

$$
P(z)=\operatorname{det}\left(z I-A-B e^{-z T}\right)
$$

where

$$
e^{-z T}=\left(\begin{array}{ccc}
e^{-z} & 0 & 0 \\
0 & e^{-2 z} & 0 \\
0 & 0 & e^{-3 z}
\end{array}\right)
$$

Let $P(z)=0$ and we obtain three solutions: $0.3155,0.6588,202433$. It is easy to find that the characteristic roots with positive real parts are located in the intersected region of a rectangle and a half circle both specified with the system

$$
\begin{gathered}
\{0 \leq \operatorname{Re}(z) \leq 3.75385,-2.40705 \leq \operatorname{Im}(z) \leq 2.40705\} \\
\cap\{(r, \theta): r \leq 3.7098,0 \leq \theta \leq 2 \pi\} .
\end{gathered}
$$

Therefore, the result shows that according to Theorem 4.4, system (4.1) is asymptotically stable outside the above region.

Example 2. Let

$$
A=\left(\begin{array}{lll}
.8462 & .6721 & .6813 \\
.5252 & .8381 & .3795 \\
.2026 & .0196 & .8318
\end{array}\right) \quad \text { and } \quad B=\left(\begin{array}{ccc}
.5028 & .3046 & .6822 \\
.7095 & .1897 & .3028 \\
.4289 & .1934 & .5417
\end{array}\right)
$$


As in Example 1, we have

$$
\begin{aligned}
\mu(A) & =16.8764, \mu(-A)=-1.8387, \mu(i A)=\mu(-i A)=3.0829,\|B\|=1.36 \\
\gamma & =17.164992>0, \beta_{0}=0.4748>0, R=17.3094
\end{aligned}
$$

Then $E_{0}=\beta, \beta$ is a root of the equation $-\mu(-A)-\|B\| \max _{1 \leq k \leq d} e^{-\beta \tau_{k}}=\beta$, $k=1,2,3$. Let $\tau_{1}=1, \tau_{2}=2, \tau_{3}=3$, then by solving the above equation, we get $\beta=1.55$. Therefore we have,

$$
E_{0}=\beta=1.55, \quad E=17.164992, \quad F_{0}=-3.371492, \quad F=3.371492 .
$$

Let $P(z)=0$ and we get the solutions $-0.988,2.765,13.482$. It is clear that the characteristic roots with positive real parts are located in the following intersected region of a rectangle and a half circle:

$$
\begin{gathered}
\{1.55 \leq \operatorname{Re}(z) \leq 17.164992,-3.371492 \leq \operatorname{Im}(z) \leq 3.371492\} \\
\cap\{(r, \theta): r \leq R, 0 \leq \theta \leq 2 \pi\} .
\end{gathered}
$$

Theorem 4.4 tells that system (4.1) is asymptotically stable outside the above intersected region.

Example 3. Let

$$
L=\left(\begin{array}{lll}
.6038 & .0153 & .9318 \\
.2722 & .7468 & .4660 \\
.1988 & .4451 & .4186
\end{array}\right), \quad M=\left(\begin{array}{lll}
.8462 & .6721 & .6813 \\
.5252 & .8381 & .3795 \\
.2026 & .0196 & .8318
\end{array}\right)
$$

and

$$
N=\left(\begin{array}{lll}
.0503 & .0305 & .0682 \\
.0709 & .0190 & .0303 \\
.0429 & .0193 & .0542
\end{array}\right)
$$

According to Section 3, Theorems 5.2 and 5.3, and Definitions 5.4 and 5.5, we get

$$
\begin{aligned}
\mu(L) & =1.36805, \mu(-L)=0.14655, \mu(i L)=\mu(-i L)=0.3885, \\
\|L\| & =1.4282,\|M\|=1.7309,\|N\|=0.136, R=3.3771, \gamma=3.596>0 \\
\beta_{0} & =-2.37455<0, E_{0}=0, E=3.596, F_{0}=-2.6165, F=2.6165 .
\end{aligned}
$$

So we have the following region that should enclose the roots with positive real parts:

$$
\{0 \leq \operatorname{Re}(z) \leq 3.596,-2.6165 \leq \operatorname{Im}(z) \leq 2.6165\} \cap\{(r, \theta): r \leq R, 0 \leq \theta \leq 2 \pi\} .
$$

Let $P(z)=\operatorname{det}\left[z I-L-M e^{-z \tau}-z N e^{-z \tau^{\prime}}\right]=0$. Taking $\tau=1, \tau^{\prime}=1.5$, we get $z=0.4977+0.1546 i ; 0.5083-0.0994 i ; 2.4072+0.1236 i$. These roots with positive real parts are all located in the above region. If we exchange the values of $\tau$ and $\tau^{\prime}$ by taking $\tau=1.5, \tau^{\prime}=1$, then all the roots have negative real parts. According to Lemma 4.1, system (5.1) is asymptotically stable. In fact, the real part $U(x, y)$ of $P(z)$ in (5.3) does not vanish on the boundary of the above region. According to Theorem 5.7, this system is asymptotically stable. 


\section{REFERENCES}

1. Driver, R. D., Ordinary and delay differential equations, Springer-Verlag, New York, 1977. MR0477368 (57:16897)

2. Hairer E., Norsett S. P. and Wanner G., Solving ordinary differential equations, SpringerVerlag, New York(1987). MR0868663 (87m:65005)

3. Desoer, C. A. and Vidyasagar, M., Feedback System: Input-Output properites, Academic Press, New York, 1977. MR0490289 (58:9636)

4. Lancaster, Peter and Tismenetsky, M., The theory of matrices, Academic Press, New York, 1985. MR0792300 (87a:15001)

5. Ioakimidis, N. I., and Anastasselou, E. G., A new, simple approach to the derivation of exact analytical formulae for the zerosof analytic functions, Appl. Math. Comput. 17(2): 123127(1985). MR0799055 (86g:30009)

6. Lu, L. H., Numerical stability of $\theta$-methods for systems of differential equations with several delays, J. CAM, 34(1991) 291-304. MR1102586 (92a:65231)

7. Hu Guang-Da and Taketomo Mitsui, Stability of linear delay differential systems with matrices having common eigenvectors, Japan J. Indust. Appl. Math., 13(1996), 487-494. MR1415066 (97i:34103)

8. Hu Guang-Di and Hu Guang-Da, Stability of discrete-delay system: boundary criteria, Applied Mathematics and Computation 80: 95-104(1996). MR1417318 (97h:34101)

9. Hu Guang-Da, Stability and numerical analysis for delay differential equations (to appear).

10. Hu Guang-Da and Mitsui, T., Stability of numerical methods for neutral delay differential equations, BIT, 35, 504-515. MR1431345 (97i:65130)

11. $\mathrm{Hu}$ Guang-Da and $\mathrm{Hu}$ Guang-Di, A relation between the weighted logarithmic norm of a matrix and the Lyapunov equation, BIT 40(2000), no.3, 606-610. MR1780410 (2001d:65064)

12. $\mathrm{Hu}$ Guang-Da and $\mathrm{Hu}$ Guang-Di, Some simple criteria for the stability of NDDEs, Appl. Math. Comput. 80(1996), no. 2-3, 257-271. MR1417326 (97h:34102)

13. $\mathrm{Hu}$ Guang-Da and $\mathrm{Hu}$ Guang-Di, Stability of neutral delay-differential system: boundary criteria, Appl. Math. Comput. 87(1997) no. 247-259. MR1468302 (98f:34110)

14. K. Dekker and J. G. Verwer, Stability of Runge-Kutta methods for stiff nonlinear differential equations, North-Holland, Amsterdam, 1984. MR0774402 (86g:65003)

15. Kuang J. X., Theory of numerical analysis for functional differential equations, Science press, 1999.

16. J. X. Kuang, J. X. Xiang, and H. J. Tian, The asymptotic stability of one-parameter methods for neutral differential equations, BIT, 34: 400-408(1994). MR1429940 (97i:65132)

17. K. Gopalsamy, Stability and oscillations in delay differential equations of population dynamics, Kluwer Academic Publishers, Boston, 1992. MR,1163190 (93c:34150)

18. D. Ya. Khusainov, and E. A. Yunkova, Investigation of the stability of linear systems of neutral type by the Lyapunov function method, Diff. Uravn, 24: 613-621(1988). MR0940744 (89e:34126)

19. V. Kolmanovskii and A. Myshkis, Applied theory of functional differential equations, Klumwer, Academic Publishers, Dordrecht, 1992. MR.1256486 (95f:34092)

20. J. K. Hale, E. F. Infante, and F.-S. P. Tsen, Stability in linear delay equations, J. Math. Anal. Appl, 105: 533-555(1985). MR0778486 (86i:34093)

21. R. K. Brayton, and R. A. Willoughby, On the numerical integration of a symmetric system of difference-differential equation of neutral type, J. Math. Analy. Appl. 18: 182-189(1967). MR 0213061 (35:3926)

22. L. M. Li, Stability of linear neutral delay-differential system, Bull, Austral Math. Soc, 38: 339-344(1988). MR0966706 (89m:34097)

23. Sun L. P., Stability of neutral delay differential equations: boundary criteria, J. Shanghai Teachers University, 30(4), 2001. MR.

College of Mathematical Sciences, Shanghai Normal University, Shanghai, 200234, People's Republic of China 